\title{
Systems of intensive vertical vortices in turbulent atmosphere
}

\author{
A. Bershadskii \\ ICAR, P.O. Box 31155, Jerusalem 91000, Israel \\ bershads@gmail.com
}

\begin{abstract}
At certain conditions a system of well-separated quasi-point vortices can appear in twodimensional turbulence. Such system contains main part (almost entire) of the flow enstrophy (mean squared vorticity). Spectral properties of the two-dimensional turbulence in the presence of the system of the quasi-point vortices have been studied using notion of the distributed chaos. Results of direct numerical simulations of decaying turbulence, of turbulence with small-scale forcing, of quasi-geostrophic turbulence with thermal damping and of turbulent thermal convection on a sphere as well as results of the Global Atmospheric Sampling Program (GASP, using commercial aircraft) measurements in the tropospheric and stratospheric turbulence over mountainous terrain (the small-scale forcing) have been used for this purpose.
\end{abstract}

\section{INTRODUCTION}

The atmospheric dynamics for spatial horizontal scales from a few kilometres up to $500 \sim 1000 \mathrm{~km}$ is often approximately horizontal. It is commonly believed that the results of the Global Atmospheric Sampling Program (GASP) measurements [1]-[3] provide a solid support for the two-dimensional approximation (it should be noted, however, that properties of quasi-two-dimensional turbulence can be rather different from those of the strictly two-dimensional one [4]). It is shown in the Ref. [3] that the atmospheric variability depends on the underlying terrain. In particular kinetic energy variances can be about six times larger over rough (mountainous) terrain than over plains and ocean. Significant difference has been also observed in the scales of the forcing. The authors of the Ref. [3] emphasize role of the small-scale forcing in quasi-two-dimensional turbulence over rough terrain of the western U.S.A. and estimate the range of scales for this forcing as $1 \sim 3 \mathrm{~km}$. On the other hand, progress in the direct numerical simulations achieved after the seminal GASP measurements revealed that isotropic homogeneous turbulence is more complex in two dimensions than in three dimensions. The two-dimensional turbulence is also more sensitive to the initial-boundary conditions and to type of forcing (cf., for instance, Refs. [5],[6],[7] and references therein). This complexity and sensitivity can be related to the abundance of the invariants for the two-dimensional motion. A remarkable phenomenon of systems of well-separated quasi-point vortices in twodimensional turbulence also was intensively numerically simulated (see for, instance, Refs. [8]-[10] and references therein). Such system contains main part (almost entire) of the flow enstrophy (mean squared vorticity $\Omega$ ) in the freely decaying isotropic homogeneous two-dimensional turbulence. Similar phenomenon is also typical for homogeneous isotropic two-dimensional turbulence with smallscale forcing [11],[12] (see Fig. 1 adapted from the Ref. [7]). The last fact is rather significant in the light of the

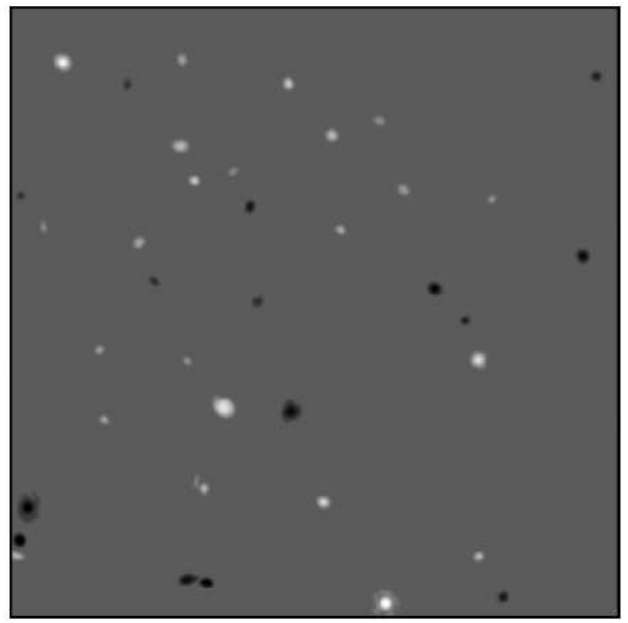

FIG. 1: Vorticity field for two-dimensional turbulence with smal-scale forcing. White and black colors are for positive and negative vorticity respectively.

GASP measurements over rough (mountainous) terrain (see above). Importance of the systems of thin intensive vertical vortices over the mountainous terrain for aviation and for short-range weather forecasting is obvious while their importance for kinetic energy spectra of the atmospheric turbulence is still obscure.

Invariants corresponding to these systems [13],[14] should be taken into account in the inertial range of scales.

\section{QUASI-POINT VORTICES AND INERTIAL RANGE OF SCALES}

While the quasi-point vortices are not too close to each other they can be considered as an adiabatically changing with time Hamiltonian system and described by cor- 


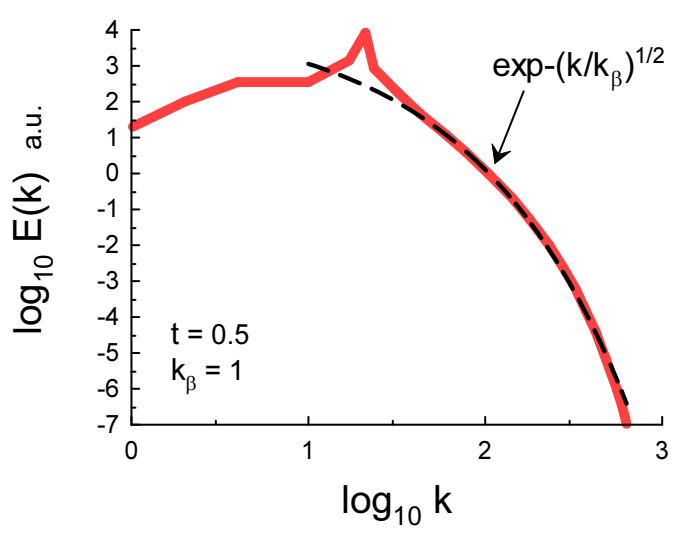

FIG. 2: Kinetic energy spectrum for decaying homogeneous isotropic two-dimensional turbulence at $t=0.5$ (the DNS time scales).

responding equations

$$
\Gamma_{i} \dot{x}_{i}=\frac{\partial H}{\partial y_{i}}, \quad \Gamma_{i} \dot{y}_{i}=-\frac{\partial H}{\partial x_{i}}
$$

with the Hamiltonian

$$
H=-\frac{1}{4 \pi} \sum_{i<j}^{N} \Gamma_{i} \Gamma_{j} \ln \left[\left(x_{i}-x_{j}\right)^{2}+\left(y_{i}-y_{j}\right)^{2}\right],
$$

where $N$ is the number of the quasi-point vortices, $\Gamma_{i}$ are their strengths (circulations), $x_{i}$ and $y_{i}$ are coordinates of their effective centres on the plane (see, for instance, Refs. [13],[15]).

The Hamiltonian system has three formal invariants on the unbounded plane

$$
P_{x}=\sum_{i} \Gamma_{i} x_{i}, \quad P_{y}=\sum_{i} \Gamma_{i} y_{i}
$$

and

$$
\mathcal{I}=\sum_{i} \Gamma_{i}\left(x_{i}^{2}+y_{i}^{2}\right)
$$

The invariants Eq. (3) are the two components of the linear momentum $\mathbf{P}$ and the invariant $\mathcal{I}$ Eq. (4) is angular momentum for the Hamiltonian system Eqs. (1-2). The Noether's theorem relates conservation of $\mathbf{P}$ and $\mathcal{I}$ to the spatial homogeneity (translational symmetry) and to spatial isotropy (rotational symmetry) correspondingly [13]. At certain conditions these (formal) invariants can be considered as adiabatic invariants for the inertial range of scales in addition to the KolmogorovObukhov adiabatic invariant $\varepsilon$ - energy dissipation rate [16]. For the two-dimensional turbulence an additional support for the adiabatic invariance of $\varepsilon$ in the inertial range of scales is provided by conservation of the enstrophy $\Omega$ for ideal incompressible fluid motions and the relationship

$$
\varepsilon=\nu \Omega
$$

where $\nu$ is viscosity.

Actually we will be interested in two combined adiabatic invariants:

$$
I_{1}=\|\mathbf{P}\| \varepsilon
$$

and

$$
I_{2}=|\mathcal{I}| \varepsilon
$$

taking into account both the quasi-point vortices and the $\varepsilon$-phenomenology in the inertial range of scales.

\section{DISTRIBUTED CHAOS APPROACH TO THE INERTIAL RANGE}

At the onset of isotropic homogeneous turbulence spectral decay of kinetic energy has exponential form [17]

$$
E(k) \propto \exp -\left(k / k_{c}\right)
$$

When the turbulence is developing the parameter $k_{c}$ becomes fluctuating and an ensemble averaging is needed in order to calculate the kinetic energy spectrum

$$
E(k) \propto \int_{0}^{\infty} P\left(k_{c}\right) \exp -\left(k / k_{c}\right) d k_{c} \propto \exp -\left(k / k_{\beta}\right)^{\beta}
$$

that uses probability distribution $P\left(k_{c}\right)$. A generalization of the exponential spectrum Eq. (8) to the stretched exponential form Eq. (9) can be also used. From the Eq. (9) one can estimate the asymptote of the $P\left(k_{c}\right)$ at large $k_{c}[18]$

$$
P\left(k_{c}\right) \propto k_{c}^{-1+\beta /[2(1-\beta)]} \exp \left(-b k_{c}^{\beta /(1-\beta)}\right)
$$

On the other hand the asymptote of $P\left(k_{c}\right)$ can be estimated from a physical consideration. Let as assume that there exists an asymptotical relationship between the characteristic velocity $v_{c}$ and the $k_{c}$ dominated by one of the adiabatic invariants Eqs. (6-7). Then from the dimensional considerations we obtain

$$
v_{c} \propto I_{1}^{1 / 4} k_{c}^{1 / 4}
$$

or

$$
v_{c} \propto I_{2}^{1 / 4} k_{c}^{1 / 2}
$$

In a general form

$$
v_{c} \propto k_{c}^{\alpha}
$$

Then for Gaussian distribution of the characteristic velocity $v_{c}$ we obtain from the Eqs. (10) and (13)

$$
\beta=\frac{2 \alpha}{1+2 \alpha}
$$


That results in

$$
E(k) \propto \exp -\left(k / k_{\beta}\right)^{1 / 3}
$$

for the Eq. (11) (domination of the spatial homogeneity over the inertial range), or in

$$
E(k) \propto \exp -\left(k / k_{\beta}\right)^{1 / 2}
$$

for the Eq. (12) (i.e. domination of the spatial isotropy over the inertial range).

\section{DIRECT NUMERICAL SIMULATIONS OF DECAYING TURBULENCE}

For decaying three-dimensional isotropic homogeneous turbulence the problem of competition between domination of the spatial isotropy (Loitsyanskii invariant [16]) and of the spatial homogeneity (Birkhoff-Saffman invariant [19]) is a long standing one. Decay of the integral characteristics of the flow can also depend on the way the turbulence is generated (see, for instance, Ref. [20] and references therein). Analogous situation takes also place for decay of the integral characteristics of two-dimensional turbulence (see, for instance, Ref. [15] and references therein).

In isotropic homogeneous turbulence isotropy dominated and homogeneity dominated attractors (i- and hattractor respectively) have different basins of attraction. The i-basin of attraction (set of the initial conditions resulting in the i-attractor) is thin and small in comparison with the h-basin (set of the initial conditions resulting in the h-attractor). Therefore, in the decaying turbulence the i-attractor approaches its fully developed state earlier than the h-attractor. However at a more advance stage of the decay the h-attractor takes its proper domination due to its larger basin of attraction.

Figure 2 shows (in the log-log scales) kinetic energy spectrum obtained in direct numerical simulations (DNS) of freely decaying homogeneous isotropic turbulence reported in Ref. [6] (the spectral data were taken from Fig. 1a of the Ref. [6] for $t=0.5$ in the DNS time scales). Random superpositions of harmonic modes with wave numbers between $\mathrm{k}=18$ and 22 were taken as initial conditions for this DNS with periodic boundary conditions. The dashed curve corresponds to the stretched exponential spectrum Eq. (16) (i.e. to the isotropy dominated distributed chaos as it was discussed above), while figure 3 shows kinetic energy spectrum obtained in the DNS for $t=1.5$. The spectral data for the Fig. 3 were taken from Fig. 4 of the Ref. [6] (ten randomly picked realizations with different random initial phases, without averaging). The dashed curve in the Fig. 3 corresponds

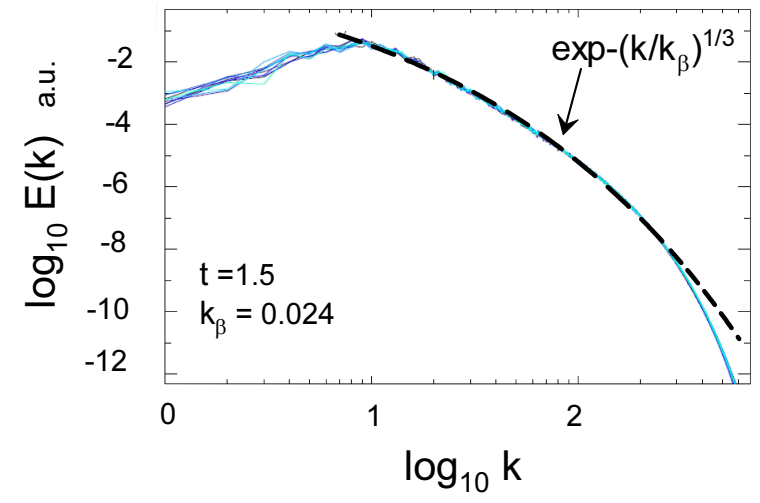

FIG. 3: Kinetic energy spectrum for decaying homogeneous isotropic two-dimensional turbulence at $t=1.5$ (ten randomly picked realizations, without averaging).

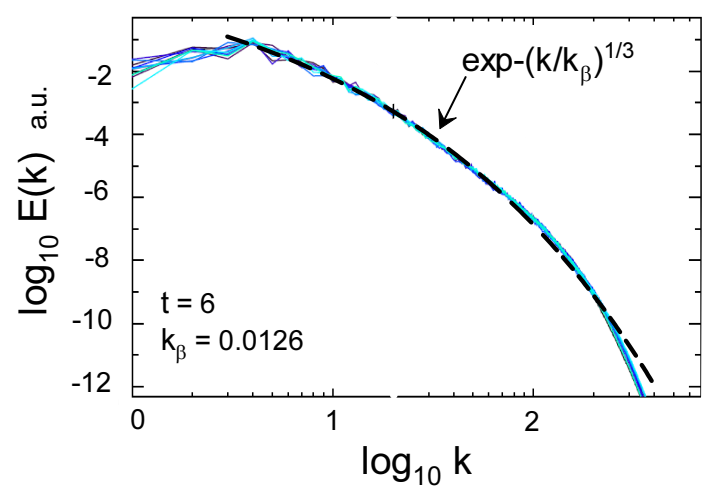

FIG. 4: As in Fig. 3 but for $\mathrm{t}=6$.

to the stretched exponential spectrum Eq. (15), i.e. to the homogeneity dominated distributed chaos as it was discussed above. Analogous spectrum has been shown in figure 4 for $t=6$ (maximal time of the DNS computations).

\section{SMALL-SCALE FORCING}

It was already mentioned in Introduction that the system of well-separated quasi-point vortices is also developing in homogeneous isotropic two-dimensional turbulence with small-scale forcing [11],[12].

In recent Ref. [7] results of direct numerical simulations with a narrow-band forcing with the characteristic forcing wavenumber $k_{f}=1024$ (see a peak in the kinetic energy spectrum shown in Fig. 5) were reported. The 


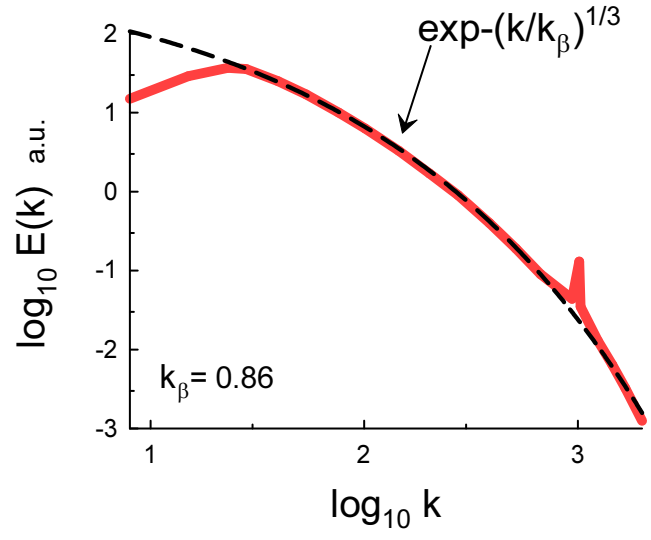

FIG. 5: Kinetic energy spectrum for the small-scale forced two-dimensional turbulence.

spectral data for the Fig. 5 were taken from Fig. 8 of the Ref. [7]. The forcing was delta-correlated in time and a hyperviscosity was applied. The initial and boundary conditions were a state of no flow and periodic respectively.

The dashed curve in the Fig. 5 corresponds to the stretched exponential spectrum Eq. (15), i.e. to the homogeneity dominated distributed chaos (cf. Figs. 3,4).

It should be noted that using their observations the authors of the Ref. [7] concluded that the energy spectrum evolves adiabatically in the inertial range of scales and "due solely to the evolution" of the quasi-point vortices population.

\section{QUASI-GEOSTROPHIC TURBULENCE WITH THERMAL DAMPING}

In recent paper Ref. [21] results of direct numerical simulations of quasi-geostrophic turbulence with smallscale forcing and large-scale thermal damping (associated with long-wave cooling in atmosphere) were reported. The authors solved numerically equations of motion:

$$
\begin{gathered}
\frac{\partial q}{\partial t}+J(\psi, q)=\alpha \lambda^{2} \psi+f \\
q=\Delta \psi-\lambda^{2} \psi
\end{gathered}
$$

where $q$ is the quasi-geostrophic potential vorticity, $\psi$ is the streamfunction, $J(\psi, q)=\partial_{x} \psi \partial_{y} q-\partial_{y} \psi \partial_{x} q$ is a Jacobian, $\lambda$ is the inverse Rossby deformation length, $\alpha$ is the thermal damping rate, $f$ is the energy input into the system (forcing). About applicability of the invariants Eqs. (3) and (4) to the quasi-geostrophic case see the recent Ref. [14].

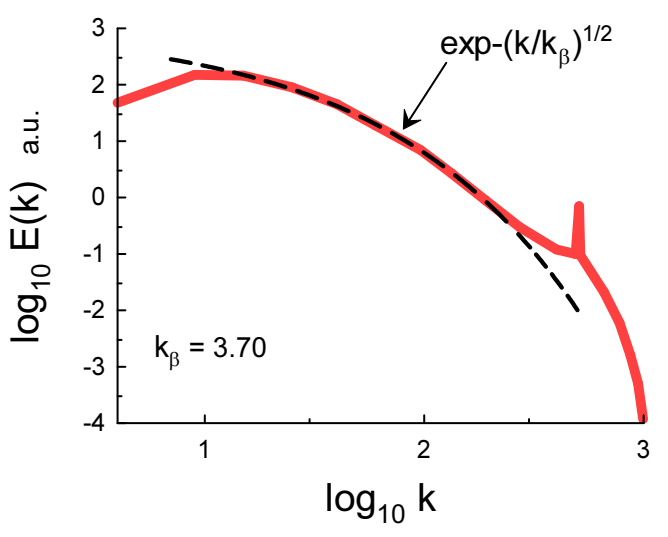

FIG. 6: Kinetic energy spectrum for the quasi-geostrophic turbulence with thermal damping for $\lambda=1$.

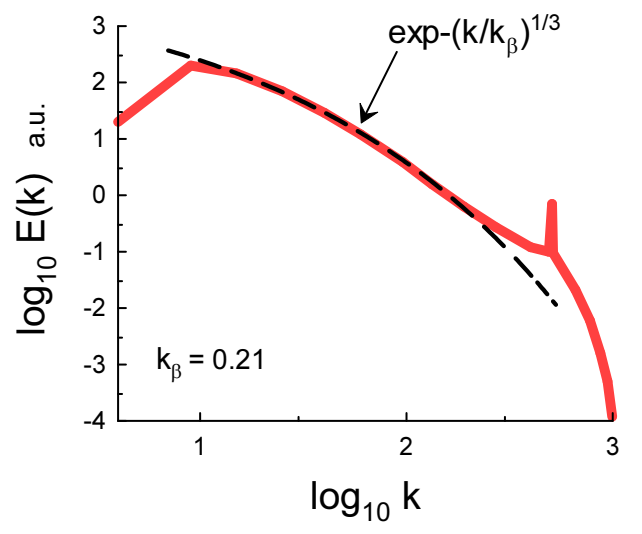

FIG. 7: As in Fig. 6 but for $\lambda=16$.

Figure 6 shows kinetic energy spectrum obtained in the direct numerical simulations reported in Ref. [21] and obtained for $k_{f}=512$ (small-scale forcing), $\alpha \lambda^{2}=10$ and $\lambda=1$ (the spectral data were taken from Fig. 6 of the Ref. [21]). The dashed curve in the Fig. 6 corresponds to the stretched exponential spectrum Eq. (16), i.e. to the isotropy dominated distributed chaos (cf. Fig. 2). Figure 7 shows kinetic energy spectrum obtained for $\lambda=16$ (the spectral data were taken from the same Fig. 6 of the Ref. [21]). The dashed curve in the Fig. 7 corresponds to the stretched exponential spectrum Eq. (15), i.e. to the homogeneity dominated distributed chaos (cf. Figs. 3,4 and 5). 


\section{THERMAL (RAYLEIGH-BÉNARD) CONVECTION ON A HEMISPHERE}

On a sphere Hamiltonian for the system of the point vortices can be written as [22]

$$
H=-\frac{1}{4 \pi R^{2}} \sum_{1 \leq j<i \leq N} \Gamma_{j} \Gamma_{i} \ln \left[2 R^{2}\left(1-\cos \gamma_{i j}\right)\right]
$$

where $R$ is radius of the sphere and $\gamma_{i j}$ are the angles between the radii vectors of the $i$ and $j$ point vortices with respect to the sphere center. Due to the Noether's theorem the rotational symmetry of the system provides three invariants

$$
\begin{gathered}
M_{1}=R \sum_{i} \Gamma_{i} \sin \theta_{i} \cos \phi_{i} \\
M_{2}=R \sum_{i} \Gamma_{i} \sin \theta_{i} \sin \phi_{i} \\
M_{3}=R \sum_{i} \Gamma_{i} \cos \theta_{i}
\end{gathered}
$$

where $\theta_{i}$ and $\phi_{i}$ are the spherical coordinates of the $i$ th vortex. These invariants commute as components of angular momentum M. From the dimensional considerations we obtain

$$
v_{c} \propto(\|\mathbf{M}\| \varepsilon)^{1 / 4} k_{c}^{1 / 4}
$$

and from the Eqs. (13-14) the power spectrum Eq. (15) for the distributed chaos.

Results of DNS of a thermal (Rayleigh-Bénard) convection on a hemisphere were reported in recent Ref. [23]. In this DNS a gradient of temperature between the heated equator and the pole creates a thermal convection with Rayleigh number $R a=10^{10}$ and Prandtl number $\operatorname{Pr}=7$.

Figure 8 shows kinetic energy spectrum obtained in the DNS (the spectral data were taken from Fig. 18 of the Ref. [23] and corresponds to a stationary state). The variable $k$ in this figure is the spherical wavenumber. The dashed curve corresponds to the stretched exponential spectrum Eq. (15).

\section{ATMOSPHERIC TURBULENCE OVER ROUGH TERRAIN}

The atmospheric variability depends on the underlying terrain. The data obtained by the seminal Global Atmospheric Sampling Program (GASP) measurements (using commercial aircraft) [3] shows that kinetic energy

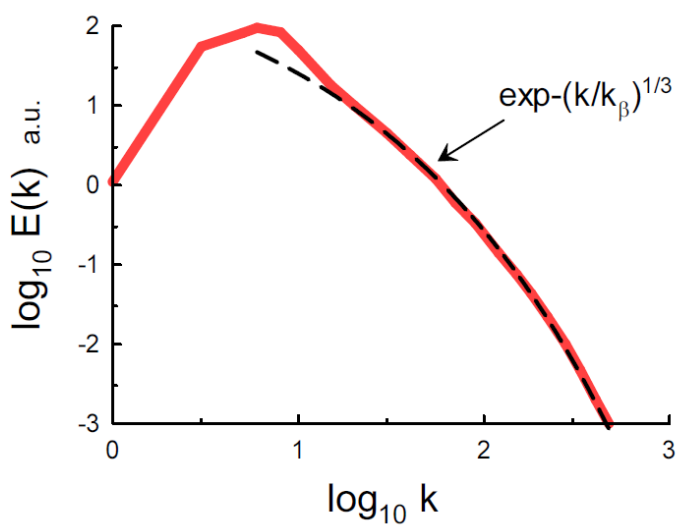

FIG. 8: Kinetic energy spectrum for a thermal (RayleighBénard) convection on a hemisphere.

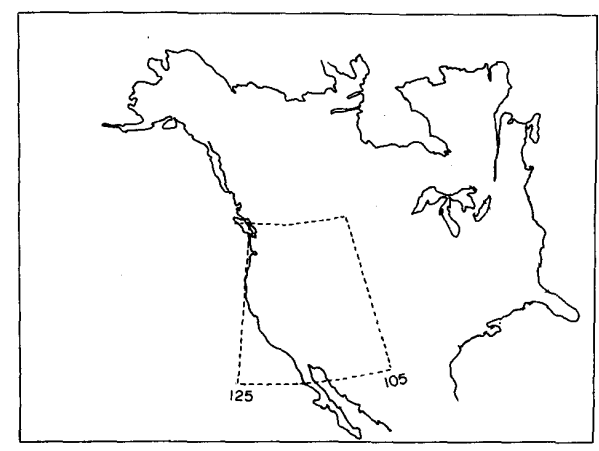

FIG. 9: Location of the GASP measurements area over the western U.S.A. (the region bounded by the dashed curves).

variances can be about six times larger over rough (mountainous) terrain than over plains and ocean. Significant difference has been also observed in the scales of the forcing. The authors of the Ref. [3] emphasize role of the small-scale forcing in quasi-two-dimensional turbulence over rough terrain of the western U.S.A. (see Fig. 9 adapted from the Ref. [3]) and estimate the range of scales for this forcing as $1-3 \mathrm{~km}$. Since we already know that inertial range in stationary homogeneous twodimensional turbulence with small-scale forcing is under strong effect of the quasi-point vortices (see Fig. 5) it is interesting to look at the kinetic energy spectra reported in the Ref. [3] for the above mentioned region of the western U.S.A..

Figures 10 and 11 show the zonal wind spectra for troposphere and stratosphere respectively (the spectral data were taken from Fig. 6 of the Ref. [3] and corresponds to high wind speed cases). The dashed curves indicate 


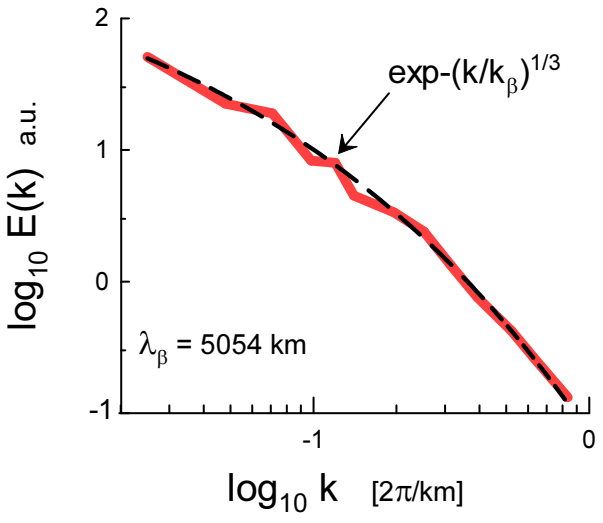

FIG. 10: Average power spectrum of zonal wind speed for high wind speed cases (troposphere).

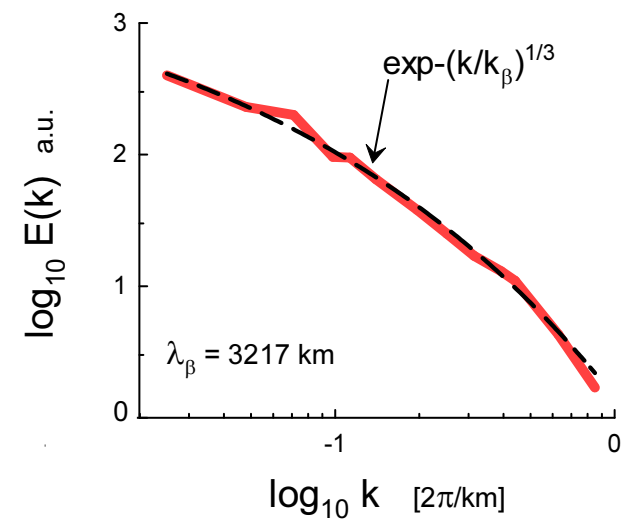

FIG. 11: As in Fig. 10 but for stratosphere.

the stretched exponential spectrum Eq. (15). The values of $\lambda_{\beta}=2 \pi / k_{\beta}$ indicate that the distributed chaos in the inertial range is tuned to the planetary waves both for troposphere and stratosphere.
[1] G.D. Nastrom and K.S. Gage, J. Atmos. Sci., 42, 950 (1985).

[2] K.S. Gage and G. D. Nastrom, J. Atmos. Sci., 43, 729 (1986)

[3] G.D. Nastrom, D.C. Fritts, and K.S. Gage, J. Atmos. Sci., 44, 3087 (1987).

[4] A. Bershadskii, E. Kit and A. Tsinober, Proc. Roy. Soc. A, 441, 147 (1993).

[5] G. Boffetta and R.E. Ecke, Ann. Rev. Fluid Mech., 44, 427 (2012).

6] P.D. Mininni and A. Pouquet, Phys. Rev. E, 87, 033002 (2013).

[7] B.H. Burgess and R.K. Scott, J. Fluid Mech., 811, 742 (2017).

[8] A. Bracco, J.C. McWilliams, G. Murante, A. Provenzale and J.B. Weiss, Phys. Fluids, 12, 2931 (2000).

[9] G.F. Carnevale, J.C. McWilliams, Y. Pomeau, J.B. Weiss and W.R. Young, Phys. Rev. Lett., 66, 2735 (1991).

[10] J.C. McWilliams, J.Fluid Mech., 219, 361 (1990).

[11] L.M. Smith and V. Yakhot, J. Fluid Mech., 274, 115 (1994).

[12] L.M. Smith and V. Yakhot, Phys. Rev. Lett., 71, 352 (1993).

[13] H. Aref, Annu. Rev. Fluid Mech., 15, 345 (1983).

[14] G. Badin and A.M. Barry, Phys. Rev. E, 98, 023110 (2018).

[15] V. Yakhot and J. Wanderer, Phys. Rev. Lett., 93, 154502 (2004).

[16] A. S. Monin, A. M. Yaglom, Statistical Fluid Mechanics, Vol. II: Mechanics of Turbulence (Dover Pub. NY, 2007).

[17] S. Khurshid, D.A. Donzis, and K.R. Sreenivasan, Phys. Rev. Fluids, 3, 082601(R) (2018).

[18] D.C. Johnston, Phys. Rev. B, 74, 184430 (2006).

[19] P.G. Saffman, Phys. Fluids, 10, 1349 (1967).

[20] P.C. Valente and J.C. Vassilicos, Phys. Lett. A., 376, 510 (2012).

[21] R.K. Scott and D.G. Dritschel, J. Fluid Mech., 721, R4 (2013).

[22] L.G. Kurakin, Chaos, 14, 592 (2004).

[23] C.-H Bruneau, P. Fischer, Y.-L. Xiong, H. Kellay, Phys. Rev. Fluids, 3, 043502 (2018). 